\title{
Linked Open Data e sustentabilidade de acervos digitais de patrimônios culturais
}

\section{Linked Open Data and sustainability of cultural heritage digital collections}

\author{
Débora Marroco Ninin | UFSCAR | debora.mninin@gmail.com
}

Ana Carolina Simionato | UFSCAR | acsimionato@ufscar.br

\begin{abstract}
Resumo: Os patrimônios culturais estão presentes em diversas instituições e prover o acesso ao seu conteúdo é extremamente necessário para a preservação de acervos digitais. Ao mesmo tempo, o gerenciamento de todas as atividades relativas à representação e acesso ao patrimônio cultural configuram-se como ações voltadas à sustentabilidade de conteúdos e serviços prestados por essas instituições culturais. Por essa razão, esse trabalho apresenta os resultados de um estudo exploratório na literatura científica nacional e internacional em que se buscou responder à questão: quais os desafios e possibilidades nas atividades de representação, organização e acesso de patrimônios culturais diante da popularização das TICs? Destacou-se o uso dos padrões de metadados para a descrição de recursos informacionais, os projetos internacionais de inclusão de dados de patrimônios culturais na iniciativa Linking Open Data, e a importância que os websites e a disponibilização de conteúdos digitais possuem para prover 0 acesso a esses patrimônios. Adentrou-se brevemente nas discussões sobre sustentabilidade e gestão do patrimônio cultural, pois se concluiu que todas essas atividades e iniciativas possuem como objetivo implícito a preservação e o uso futuro dos conteúdos relativos ao patrimônio cultural.

Palavras-chave: Organização e representação; Patrimônio cultural; Sustentabilidade; Linked Open Data; Tecnologias de informação e comunicação.
\end{abstract}

Abstract: Cultural heritage are part of many institutions and providing access to its content is extremely necessary to digital collections preservation. At the same time, the management of all activities related to representation and access to cultural heritage are indeed actions directed to sustainability of contents and services provided by these institutions. Therefore, this work presents the results of an exploratory study in the national and international scientific literature that pursued to answer the question: what are de challenges and possibilities in the activities of representation, organization and access of cultural heritage with the popularization of the information technologies? The use of standards metadata for description, the initiatives of inclusion of cultural heritage in the linked open data movement, the importance of the web sites and the provision of digital content to the user's access was discussed. Also, was briefly discussed considerations about sustainability and management of cultural heritage, because was concluded the all the activities and movements discussed has as goal the preservation and future uses of the content related to cultural heritage. Keywords: Organization and representation; Cultural heritage; Sustainability; Linked Open Data; Information and Communication Technologies. 


\section{Introdução}

O protagonismo das novas tecnologias no desenvolvimento da sociedade moderna envolve a sua distribuição entre os estudos científicos e os setores de mercado, o que nem sempre é feito de forma igualitária. Por diversos motivos, as novas tecnologias e a sua relação com as Ciências Humanas e Sociais Aplicadas recentemente têm-se destacado pela aplicação das mesmas em suas práticas. Diante de possibilidades cada vez mais sofisticadas de desenvolvimento e uso das novas tecnologias de informação e comunicação (TIC), também é esperado alcançar outras formas de contribuições que podem ser realizadas em relação à denominada Humanidades Digitais (SANTAREM SEGUNDO, 2015), mais especificamente em relação à representação, organização e acesso ao conteúdo de patrimônios culturais. Dessa forma, esse trabalho apresenta resultados de um estudo exploratório na literatura científica nacional e internacional sobre a temática em discussão. Pelo método perspectivista (PETERSON, 1996) busca-se responder à questão: quais os desafios e possibilidades nas atividades de representação, organização e acesso à patrimônios culturais diante das novas possibilidades tecnológicas?

No intuito de responder o questionamento, esse trabalho objetiva-se a apresentar o uso de padrões para descrição de recursos informacionais e o crescimento do movimento de abertura e ligação dos dados (Linked Open Data), das formas de disseminação de informações, bem como algumas considerações relativas à sustentabilidade de produtos e serviços digitais. É importante esclarecer que os assuntos aqui tratados são complementares entre si. Por essa razão, em alguns momentos do trabalho, certas temáticas possuem relações estreitas que não permitem a plena separação ou supressão de suas partes.

\section{Patrimônio cultural na era da informação}

Primeiramente, apresenta-se uma definição de patrimônio cultural, suas especificidades e sua contextualização atual, tanto no cenário nacional quanto no internacional. Quando se fala em patrimônio, também denominado como herança 
cultural, naturalmente remete-se aos museus e aos seus vastos e valiosos acervos. De fato, essas instituições sempre desempenharam um papel fundamental na preservação e difusão dos objetos considerados essenciais para a história da humanidade. Discute-se na área de Ciência da Informação, ainda que timidamente no Brasil (SOUZA, CRIPPA, 2010) os temas sobre patrimônio cultural, e como e quais as contribuições que a Biblioteconomia e a Arquivologia oferecem ao campo da Museologia e vice-versa, uma vez que autores como Dahlström, Hansson e Kjellman (2012) e Maroevic (1998) consideram os arquivos e os acervos bibliográficos também como passíveis de configurarem como patrimônios culturais: arquivos, assim como museus, lidam com objetos únicos, enquanto que as bibliotecas têm como objetivo reunir o conhecimento humano (DAHLSTRÖM, HANSSON, KJELLMAN, 2012).

Dentre as definições de patrimônio encontradas na literatura, destaca-se a de Hyvönen (2012, p. 01, grifo e tradução nossa[1]):

Patrimônio cultural refere-se ao legado de objetos físicos, ambientes, tradições e conhecimentos de uma sociedade, que pertencem ao passado, que são mantidos e desenvolvidos no presente e que são preservados (conservados) para o benefício das gerações futuras.

A partir desta afirmativa podem-se especificar as características materiais e imateriais do patrimônio. O Conselho Internacional de Museu - ICOM (1999) define patrimônio como expressão material e/ou espiritual que caracteriza determinado povo ou comunidade. Da mesma forma, Maroevic (1998) relembra a divisão do patrimônio material entre móvel e imóvel, o que remete novamente a Hyvönen (2012) que se utiliza da subdivisão do patrimônio entre tangível (objetos, artefatos, livros, etc.), intangível (linguagem, tradições, folclore, etc.) e natural (lugares, biodiversidade, etc.). Note-se que a sobreposição dessas características é possível, uma vez que patrimônios naturais podem ser móveis (pedras e fósseis de animais, por exemplo) ou imóveis.

Quando se trata deste assunto no Brasil, Souza e Crippa (2010) lembram que as questões sobre as características do patrimônio brasileiro e suas influências para a identidade nacional começaram a ser questionadas somente a partir da década de 
20, dentro do Movimento Modernista Brasileiro. Os autores ainda trazem o escopo de patrimônio dado pelo artigo 216 da Constituição Federal de 1988, ainda vigente:

\begin{abstract}
Constituem patrimônio cultural brasileiro os bens de natureza material e imaterial, tomados individualmente ou em conjunto, portadores de referência à identidade, à ação, à memória dos diferentes grupos formadores da sociedade brasileira, nos quais se incluem: I - as formas de expressão; II - os modos de criar, fazer e viver; III - as criações científicas, artísticas e tecnológicas; IV - as obras, objetos, documentos, edificações e demais espaços destinados às manifestações artístico-culturais; $V$ - os conjuntos urbanos e sítios de valor histórico, paisagístico, artístico, arqueológico, paleontológico, ecológico e científico. (BRASIL, 1988)
\end{abstract}

Esta definição mostra-se de acordo com Dahlström, Hansson e Kjellman (2012) e Maroevic (1998) de que tanto museus quanto arquivos e bibliotecas efetivamente possuem patrimônios culturais como objetos de salvaguarda. Souza e Crippa (2010) atribuem ao patrimônio o valor documental, e por isso, é considerado uma fonte de informação - afirmativa compartilhada pelos próprios Dahlström, Hansson e Kjellman (2012) quando estes tratam da digitalização desses objetos, assunto a ser abordado nas próximas seções do trabalho. Nesse sentido, Maroevic (1998, p.136, tradução nossa [2]) atenta para as influências do espaço, do tempo e da própria sociedade sobre o patrimônio: "Na realidade é a harmonia entre matéria, forma e significado, que se manifesta no tempo, no espaço e na sociedade, que faz patrimônios culturais individuais autênticos".

Falar em patrimônio, portanto, é falar em forma e conteúdo, em permanências e mudanças. Todas essas nuances devem ser identificadas pelos profissionais - curadores, arquivistas ou bibliotecários - que lidam com este tipo de acervo.

\title{
2.1. Gestão do patrimônio cultural
}

As considerações apontadas até o momento levam à necessidade de uma breve discussão sobre o papel das instituições - museus, arquivos e bibliotecas sobre a gestão do patrimônio cultural sob sua responsabilidade. Como já apontado, embora tenham características diferenciadas de modos de atuação, todas essas 
instituições têm como objetivo final e coletivo a preservação e disseminação de patrimônios culturais (DAHLSTRÖM, HANSSON, KJELLMAN, 2012).

Nesse sentido, o ICOM (1999) coloca as instituições museológicas em específico como espaço e meio de comunicação e instrumento de educação permanente, com foco em ações democráticas e plurais. Ao mesmo tempo, defende que museus devem orientar seu discurso para o presente, focando aos processos culturais - dessa forma, o processo seria mais importante do que o próprio objeto cultural. Em sentido mais amplo, Dahlström, Hansson e Kjellman (2012) afirmam que as tomadas de decisão das instituições influenciam no modo como o patrimônio é exposto para o público, e consequentemente, direciona o uso que a comunidade fará desse conteúdo exposto.

A questão sobre a autenticidade do patrimônio apontada por Maroevic (1998) também é retomada, uma vez que para este autor os significados atribuídos e as mudanças materiais e espirituais de uma sociedade determinam a autenticidade de um patrimônio mais do que sua origem.

Hyvönen (2012) aponta como principal desafio para o campo de estudo sobre patrimônio cultural as questões de múltiplos formatos, tópicos, línguas e linguagens, culturas e públicos-alvo (comunidades leigas e experts) que a sociedade da informação proporciona via suas tecnologias.

\section{Representação de recursos informacionais na Web}

A partir do contexto da gestão de acervos condicionados ao patrimônio cultural, as atividades de organização e recuperação da informação são caracterizadas como o cerne da Ciência da Informação, tendo sido inclusive o pilar de sua formação como ciência (SILVA, FREIRE, 2012; SOUZA, CRIPPA, 2010). Note-se que a recuperação, acesso e reuso da informação é tido como processo final de um ciclo de atividades anteriores, especialmente as atividades de representação e da própria organização. Organizar e representar, contudo, não devem ser atividades confundidas entre si. Glushko (2013) diferencia as atividades de organização de objetos e organização de informações sobre objetos, de forma 
que esta última se aproxima muito mais das atividades de representação de recursos do que as de organização física do recurso em si. O termo objeto informacional, ou recurso informacional, tem sido amplamente utilizado em detrimento do termo documento quando se fala em representação da informação, por ser um termo com maior abrangência sobre o que pode ser considerado informação ou objeto informativo. Gilliland (2008) caracteriza o recurso informacional como sendo constituído de conteúdo, contexto e estrutura, de forma que aqui se reforça a ideia de matéria, forma e significado do documento de patrimônio, como também apresenta Maroevic (1998).

Nesse sentido, o crescimento e a popularização da TIC nas últimas décadas proporcionaram uma verdadeira revolução do que diz respeito à atividade de representação da informação. Mais do que automatizar o trabalho e proporcionar uma economia de espaço físico, isso é, um computador ocupa menos metros quadrados do que um catálogo de fichas ou mesmo algumas estantes de acervo físico, o uso de ferramentas TIC permitiram um salto qualitativo na padronização e colaboração do processo de representação. Muito se tem discutido na área de Ciência da Informação, sobre a adoção de padrões de representação, voltados às necessidades de cada tipo de acervo, de forma a permitir a interoperabilidade e a cooperação entre os sistemas e a melhor preservação digital das informações (ANDRADE, 2010), como pode ser observado na Tabela 1 (Tabela 1, em apêndice). Ressalva-se que alguns padrões são estruturados e ainda vigentes antes mesmo da invenção dos computadores. Representar os recursos em formatos digitais ainda possibilita o acesso remoto a esses recursos, o que por si só já atesta o poder de disseminação proporcionado pelas TIC: um usuário brasileiro pode acessar pela tela de seu computador pessoal um catálogo de uma biblioteca que se encontra no Japão, por exemplo.

Inserido a essas discussões, os metadados são os elementos 'chave' de todo o ciclo informacional. Sua definição condiz a "[...] atributos essenciais de um objeto informativo" (GILLILAND, 2008, p. 22) e, portanto, têm como objetivo descrever um recurso informacional (GILLILAND, 2008). Dessa forma, as atividades de 
representação, mesmo nos meios analógicos, sempre se destacaram pela importância da definição de quais metadados seriam necessários para a descrição de um recurso. É útil, porém, diferenciar os conceitos de dados e de metadados: o primeiro se constitui do metadado acrescido do valor do recurso - se o título de uma obra, por exemplo, é Dom Casmurro, o que se tem é um dado (metadado + valor), sendo o atributo 'título' isoladamente, o metadado. Ou seja, não basta trazer a tona discussões sobre a padronização dos metadados em si, mas sim de sua estrutura, valores e conteúdo (GILLILAND, 2008).

A Tabela 1 também apresenta os formatos de dados e padrões de intercâmbio, fundamentais para que se trabalhe com sistemas interoperáveis. É notável o uso da Extended Markup Language (XML) como a principal linguagem de marcação, uma vez que esta “[...] não é uma linguagem de marcação predefinida (como o HTML) e possibilita ao autor do documento projetar sua própria marcação" (ALMEIDA, 2002). Seu uso tem sido incentivado justamente por ser uma linguagem simples e versátil, além de fazer parte do rol de ferramentas recomendadas pelo World Wide Web Consortium (W3C) para inclusão de ferramentas no projeto Web Semântica WORLD WIDE WEB CONSORTIUM, 2015) e mais especificamente, no movimento linked open data.

Outro aspecto importante da representação de informações em relação às TIC reside nas tecnologias envolvidas na digitalização de acervos de patrimônios culturais. A produção de imagens relativas a determinado recurso, além de ser uma representação em si, atua como mediadora entre o recurso e o usuário (DAHLSTRÖM, HANSSON, KJELLMAN, 2012). Estes autores situam a digitalização como uma atividade de organização do conhecimento, pois, contextualiza, descontextualiza ou mesmo fornece outra contextualização aos patrimônios culturais de acordo com o tipo de digitalização escolhida: em massa ou crítica, cujas características são comparadas na Tabela 2 (Tabela 2, em apêndice).

Os processos de representação de patrimônios culturais envolvem as escolhas tomadas pela instituição no que diz respeito aos padrões e ferramentas a serem utilizados na descrição e aos procedimentos de digitalização dos recursos 
representados. Sistematicamente, essas decisões vão impactar o acesso, a disseminação e o uso dos conteúdos pelos usuários e exigirão outras medidas da instituição para a garantia de sustentabilidade de seus produtos e serviços.

Visto a importância da representação e organização dos recursos informacionais, caracteriza-se a potencial contribuição que esses processos informacionais direcionam para novas discussões e campos de atuação, como exemplo o gerenciamento de dados.

Os dados podem ser originários e derivados de vários contextos, como o campo acadêmico, fruto de pesquisas, produzidos por processos gerenciais, advindos de realizações comerciais e tecnológicas, ou até mesmo, são dados de catálogos, originárias da descrição e registro de um acervo - como o foco desse trabalho, os acervos culturais.

Mas para que esses dados sejam publicados na Web, há uma série de princípios que devem ser obedecidos para que esses dados ou conjunto de dados (datasets) sejam acessados e reutilizados, e nesse ponto, ressalta-se novamente a importância do tratamento informacional dessas unidades.

\subsection{Linked open data}

Adentro do movimento crescente da publicação de dados na Web, os princípios Open Data caracterizam que a publicação deve ser aberta, ou seja, os dados devem seguir padrões e formatos que o caracterizem como abertos.

Nesse viés, outra iniciativa atrelada às políticas de dados abertos, é denominada como Linking Open Data (LOD), que além de apontar os princípios de formato aberto, sugere políticas de como esses dados podem se conectar. O Linking Open Data é a denominação utilizada para

[...] um conjunto de melhores práticas para publicação de dados estruturados na Web, permitindo estabelecer links entre itens de diferentes fontes de dados para formar um único espaço de dados global (SANTAREM SEGUNDO, 2015, p. 225).

Nessa perspectiva, a evolução dos registros informacionais em bibliotecas, arquivos e museus seria a desvinculação de seus dados dos catálogos para 
alcançarem uma rede aberta e ampla, pois a publicação de dados torna-os independentes dos sistemas de gerenciamento de catálogos e ao mesmo tempo permite um novo paradigma de interligação de acervos no ambiente digital (MARCONDES, 2016). A iniciativa Linking Open Data também é vinculada ao movimento Linked Open Data defendido pela própria W3C, que além de incentivar o uso do XML, ainda explora, desenvolve e recomenda outras ferramentas e procedimentos para melhorar a experiência de uso e desenvolvimento da Web.

O movimento Linked Open Data destaca o uso do Resource Description Framework (RDF), responsável pela ligação dos dados, e das ontologias, que inclui os vocabulários gerais ou de domínio. Tim Berners-Lee, considerado o criador da Web, gestor do W3C e criador dos princípios Linked Data e, posteriormente, do movimento Linked Open Data, elaborou um ranking de avaliação da qualidade dos dados publicados e suas ligações, chamado de Five Star Linked Open Data (BERNERS-LEE, 2006).

Arakaki (2016) reúne diversas iniciativas institucionais de publicação de dados de patrimônios culturais em andamento no cenário internacional, destacando-se aqui os movimentos Linked Open Data in Libraries, Archives and Museums (LODLAM), Linked Open Data Galleries, Libraries, Archives and Museums (LODGLAM), Open Galleries, Libraries, Archives and Museum (Open GLAM) e o acervo da Europeana. No mesmo sentido, Thorsen e Pattuelli (2016) destacam a Digital Public Library of America, o Social Networks and Archival Context Project, o regesta.exe da Itália, a publicação de dados do Amsterdan Museum, e a ligação de dados internos do Smithsonian Museum of American Art como outros exemplos de iniciativas dentro do movimento Linked Open Data, ao mesmo tempo em que discutem seu próprio projeto, o Linked Jazz. O ranking de Berners-Lee foi adaptado, em 2011, por membros da iniciativa LODLAM para abarcar as especificidades dos dados de patrimônios culturais: o 4-Star Classification-Scheme for Linked Open Cultural Metadata (VOSS, 2012; SMITH, 2011). 
A inserção de dados sobre patrimônios culturais na iniciativa Linking Open Data, bem como os esforços voltados especificamente para a publicação desse tipo de dado justificam-se pela criação de novos serviços, pela construção de parcerias entre instituições que não necessariamente precisam pertencer ao mesmo país, mas principalmente, pelos novos conhecimentos que só podem ser alcançados com dados abertos e disponíveis para manipulação e uso (SMITH, 2011). Marcondes (2011) discute particularmente esta última questão quando explica que as ligações entre os dados de patrimônios culturais não possuem necessariamente equivalência semântica, mas são culturalmente significativas ou mesmo indiretas:

Falar em relações culturalmente significativas entre objetos arquivísticos, bibliográficos e museológicos pertencentes a diferentes acervos significa reconhecer o valor e o potencial cultural destas relações. É um trabalho de curadoria, naturalmente dentro de um novo contexto (MARCONDES, 2011, p.75, grifo do autor).

Portanto, publicar dados de patrimônios culturais se configura como uma importante forma de agregação de valor aos acervos museológicos, arquivísticos e bibliotecários, ao mesmo tempo em que permitem uma nova forma de visualização e acesso a esses patrimônios, assuntos que serão abordados em seguida.

\section{Acesso ao acervo de patrimônio cultural}

Embora os processos de representação de recursos informacionais sejam fundamentais para a recuperação da informação, sendo, por isso, amplamente abordados pela Ciência da Informação, por si só não são suficientes para garantir o acesso aos conteúdos produzidos. Para Maron, Yun e Pickle (2013, p.44) as instituições ainda têm que lidar com o desafio de desenvolvimento de métodos de disseminação de seus acervos e produção de impacto social no seu ambiente de atuação. Para Santos (2013), os desafios atuais não residem na representação, mas sim na apresentação do conteúdo representado. Da mesma forma Jardim (1999, p. 72) esclarece que 
[...] o termo acesso relaciona-se a um direito, mas também a dispositivos que o viabilizem, ou seja, com conjunto de procedimentos e condições materiais que permitam o exercício efetivo desse direito.

Dentre este conjunto de dispositivos, este trabalho identificou como porta de entrada de instituições de guarda de patrimônios culturais no ambiente digital os websites, que nas palavras de Maron, Yun e Pickle (2013) estão chegando ao ponto de se tornarem um fim em si mesmos, com objetivos maiores do que a simples disponibilização online de seus catálogos: são pontes de relacionamento entre a instituição e os usuários, plataformas de compartilhamento de conhecimento.

Nesse sentido, Terso (2010) centraliza a questão do acesso à necessidade de alfabetização informacional, para que as instituições abandonem os processos de aprendizado passivo praticados até o momento e se aproximem de processos mais dinâmicos, como os propostos pelo ICOM (1999). Ao mesmo tempo, Burnette et al. (2009) conduziu um estudo sobre a necessidade de reconfiguração de websites de museus, apresentando como resultados uma lista de recomendações para instituições que sintam necessidade dessa reconfiguração. Dentre elas, destaca-se a necessidade de que as instituições reconheçam nos seus websites sua identidade institucional (missão, visão e valores) e as diferenças que podem existir entre os usuários que frequentam fisicamente a instituição e aqueles que apenas se utilizam de suas plataformas digitais. Outra recomendação diz respeito ao fato de que o lançamento de um website não se constitui em um produto final, mas sim em um conjunto de conteúdo a ser periodicamente modificado, o que leva às discussões sobre sustentabilidade de ações, procedimentos, serviços e conteúdos relativos ao patrimônio cultural.

Independentemente dos objetivos e procedimentos adotados pelas instituições para a gestão do patrimônio cultural, a preocupação com a sustentabilidade dos conteúdos digitais gerados deve ser prioridade, pois esta não depende apenas da preservação desses conteúdos para o futuro, mas também do seu acesso e uso pelos usuários, bem como a geração de impacto na comunidade (MARON, YUN, PICKLE, 2013). Estes autores dividem os conteúdos digitais em 
duas categorias, de acordo com o tipo de gestão que exigem: os que requerem manutenção com determinada periodicidade ou aqueles que devem ser radicalmente modificados. A sustentabilidade é, então, uma atividade contínua e está além do processo de curadoria digital. Envolve propriedades que as instituições devem ser capazes de identificar e a partir de então, alinhar seus procedimentos e tomadas de decisões a partir dessas características. Para a Council on Library and Information Resources (2001, p.19, tradução nossa[3]):

Entre os elementos necessários para a sustentabilidade de programas digitais de instituições de todos os tamanhos estão os padrões e as melhores práticas, arquiteturas digitais comuns e coerentes e meios práticos de criação e compartilhamento de uma base de conhecimentos.

O que se percebe nesta afirmação é a recorrência da ideia de que a sustentabilidade não se prende às questões de preservação, mas engloba todo o ciclo de gerenciamento informacional, incluindo os processos de organização, representação, recuperação, acesso e uso dos conteúdos digitais.

Esta é a principal razão pela qual se afirmou inicialmente que os assuntos aqui discutidos são dependentes entre si: para que se tenham ações sustentáveis é necessário que se olhe para as ações da instituição como um todo e de forma sistêmica - não há como disseminar e prover acesso a um conteúdo que não está devidamente representado, da mesma forma que não há razão de se representar algo que não será mostrado e disseminado.

\section{Considerações finais}

As discussões apresentadas nesse trabalho, sobre a adoção de padrões de representação de conteúdo, a publicação de dados pelo movimento Linked Open Data e as novas formas de comunicação entre instituição e público, notadamente os websites e os catálogos, configuram-se como ações voltadas à sustentabilidade e, por isso, são desafios a serem superados com o uso intensivo das TIC.

Estas são ao mesmo tempo fonte de soluções e de novos desafios, exigindo grandes esforços dos profissionais da informação na sua aplicação. Embora, o trabalho apresente nesse tópico considerações, é destacado que este se trata de um 
estudo ainda inicial para as inúmeras possibilidades para catálogos, acervos de patrimônio cultural e a iniciativa Linking Open Data.

O trabalho elucida sobre a temática por meio de um levantamento bibliográfico nacional e internacional. A partir desse levantamento, houve um destaque para o uso de padrões de metadados na descrição de recursos informacionais, as iniciativas internacionais de inclusão desse tipo de acervo no Linking Open Data, e a importância que os websites e a disponibilização de conteúdos digitais possuem para prover o acesso a esses patrimônios.

Consequentemente, adentrou-se nas discussões sobre sustentabilidade e gestão do patrimônio cultural, visto que se conclui que todas essas atividades e iniciativas possuem como objetivo implícito: a preservação e o uso futuro dos conteúdos relativos ao patrimônio cultural. Em relação ao Brasil, por meio da literatura, destaca-se a falta de iniciativas nas instituições que trabalham com 0 patrimônio cultural para a abertura de seus dados, fato que prejudica a visibilidade e o acesso ao patrimônio cultural nacional dentro e fora do país. Em contrapartida, as iniciativas internacionais ganham cada vez mais espaço, visto a Europeana, a Digital Public Library of America e as demais iniciativas apontadas nesse trabalho. Espera-se que este estudo sirva de apoio para cientistas e profissionais da informação no desenvolvimento de novos estudos, tecnologias, ferramentas, produtos e serviços relativos ao patrimônio cultural, tanto em museus, quanto em bibliotecas e arquivos.

\section{Notas}

[1] "Cultural Heritage $(\mathrm{CH})$ refers to the legacy of physical objects, environment, traditions, and knowledge of a society that are inherited from the past, maintained and developed further in the present, and preserved (conserved) for the benefit of future generations" (HYVÖNEN, 2012, p. 01)

[2] "It is actually the concord of material, form and significance, which is manifested in time, space and society, that makes individual parts of cultural heritage authentic" (MAROEVIC, 1998, p.136)

[3]"Among the elements needed by institutions of all sizes for sustainable digital programs are standards and best practices, coherent and common digital 
architectures, and ongoing means for creating and sharing a knowledge base" (COUNCIL ON LIBRARY AND INFORMATION RESOURCEs, 2001, p.19)

\section{Referências}

ALMEIDA, Maurício Barcellos. Uma introdução ao XML, sua utilização na Internet e alguns conceitos complementares. Ciência da informação, v. 31, n. 2, p. 5-13, 2002. Disponível em:

<http://www.scielo.br/scielo.php?pid=s0100-

$19652002000200001 \&$ script $=$ sci_abstract\&tlng=pt >. Acesso em 24 jan. 2017

ALVES, Rachel Cristina Vesú. Metadados como elementos do processo de catalogação. 2010. (Doutorado em Ciência da Informação) - Faculdade de Filosofia e Ciências, Universidade Estadual Paulista, Marília, 2010.

ANDRADE, Ricardo Sodré. Aspectos introdutórios da representação de informação arquivística: a Norma Brasileira de Descrição Arquivística (NOBRADE), a Descrição Arquivística Codificada (EAD-DTD) e o Projeto Archives Hub. In: SILVA, Rubens Ribeiro Gonçalves da et al (Org). Cultura, representação e informação digital. Salvador: Edufba, 2010. p.185 - 210.

ARAKAKI, Felipe Augusto. Linked Data: ligação de dados bibliográficos. 2016. 146 f. Dissertação (Mestrado em Ciência da Informação) - Universidade Estadual Paulista, Marília, 2016.2 Disponível em: <http://repositorio.unesp.br/bitstream/handle/11449/147979/arakaki_fa_me_mar.p $\mathrm{df}$ ? sequence $=2$ \&isAllowed $=\mathrm{y}>$. Acesso em: 12 jan. 2017

BERNERS-LEE, Tim. Linked Data: design issues. 2006. Disponível em: <http://www.w3.org/Designlssues/LinkedData.html>. Acesso em 13 nov. 2106.

BRASIL. Constituição (1988). Disponível em: <http://www.planalto.gov.br/ccivil_03/constituicao/constituicao.htm>. Acesso em: 17 de mar. 2017.

BURNETTE, Allegra et al. Redesigning your museum's web site: a survivors' guide. In: MUSEUMS AND THE WEB, Indianapolis, USA, 2009. Anais... Toronto: Archives \& Museum $\quad$ Informatics, 2009.2 Disponível em $<w w w$.archimuse.com/mw2009/papers/burnette/burnette.html>. Acesso em: 10 mar. 2017.

CASTRO, Fabiano Ferreira de. Padrões de representação e descrição de recursos informacionais em bibliotecas digitais na perspectiva da ciência da informação: Uma abordagem do MarcOnt initiative na era da web semântica. 2008. 201f. Dissertação (Mestrado em Ciência da Informação) - Faculdade de Filosofia e Ciências, Universidade Estadual Paulista, Marília, 2008. 
CONSELHO INTERNACIONAL DE MUSEU. Declaração de Caracas (1992). Cadernos de Museologia, n.15, 1999, p. $243-265$.

COUNCIL ON LIBRARY AND INFORMATION RESOURCES. Building and sustaining digital collections: models for libraries and museums. Washington, DC: Council on Library and Information Resources, 2001. Disponível em: <www.clir.org/pubs/reports/pub100/pub100.pdf>. Acesso em: 13 mar. 2017.

DAHLSTRÖM, Mats. Critical editing and critical digitization. In: THOUTENHOOFD, Ernst; WEEL, Adriaan van der; PEURSEN, Wido (Eds.). Text comparison and digital creativity. Amsterdam: Brill, 2010, p. 79-97.

DAHLSTRÖM, Mats; HANSSON, Joacim; KJELLMAN, Ulrika. As we may digitize: institutions and documents reconfigured". Liber Quarterly, v. 21, n.3-4, abr. 2012 , p. 455-474. Disponível em: <https://www.liberquarterly.eu/articles/10.18352/lq.8036/>. Acesso em 14 mar. 2017.

GILL, Tony. Metadata and the Web. In: BACA, Murtha (Ed.). Introduction to metadata. 2. ed. Los Angeles: The Getty Research Institute, 2008. p.20-37.

GILLILAND, Anne. Setting the stage. In: BACA, Murtha (Ed.). Introduction to metadata. 2. ed. Los Angeles: The Getty Research Institute, 2008. p.1-19.

GLUSHKO, Robert J. (Ed.). The discipline of organizing. Massachusetts: MIT Press, 2013.

HOLLÓS, Adriana Cox. Preservação e memória social. In: SILVA, Rubens Ribeiro Gonçalves da et al (Org). Cultura, representação e informação digital. Salvador: Edufba, 2010. p. 29-40.

HYVÖNEN, Eero. Publishing and using cultural heritage linked data on the Semantic Web. EUA: Morgan \& Claypool, 2012 (Synthesis lectures on Semantic Web: theory and technology, n. 3).

JARIDM, José Maria. Transparência e opacidade do Estado no Brasil: usos e desusos da informação governamental. Niterói: EdUFF, 1999. 239 p.

MARCONDES, Carlos Henrique. Interoperabilidade entre acervos digitais de arquivos, bibliotecas e museus: potencialidades das tecnologias de dados abertos interligados. Perspectivas em Ciência da Informação, v. 21, n. 2, p. 61-83, abr./jun. 2016. Disponível em: <http://portaldeperiodicos.eci.ufmg.br/index.php/pci/article/view/2735>. Acesso em: 19 jan. 2017 
MARCONDES, Carlos Henrique. Representação e economia da informação. Ciência da Informação, Brasília, v. 30, n. 1, p. 61-70, jan./abr. 2001.

MAROEVIC, Ivo. The phenomenon of cultural Heritage and the definition of a unit of material. Nordisk museologi, online, n.2, 1998, p.135-142. Disponível em: <https://www.journals.uio.no/index.php/museolog/article/view/3829>. Acesso em 14 mar. 2017.

MARON, Nancy L.; YUN, Jason; PICKLE, Sarah. Sustaining our digital future: institutional strategies for digital content. New York: Ithaka S+R, 2013.

PETERSON, Donald (Org). Forms of representation: an interdiciplinary theme for cognitive science. Wiltshire: Cromwell Press, 1996.

SANTAREM SEGUNDO, José Eduardo. Web semântica, dados ligados e dados abertos: uma visão dos desafios do Brasil frente às iniciativas internacionais. In: ENCONTRO NACIONAL DE PESQUISA EM CIÊNCIA DA INFORMAÇÃO, 16, 2015, João Pessoa. Anais... João Pessoa, Universidade Federal da Paraíba, 2015.

SANTOS, Plácida L. V. Amorim da Costa. Catalogação, formas de representação e construções mentais. Tendências da Pesquisa Brasileira em Ciência da Informação, v. 6, n.1, 2013.

SANTOS, Plácida L. V. Amorim da Costa; SANT'ANA, Ricardo César Gonçalves. Dado e Granularidade na perspectiva da Informação e Tecnologia: uma interpretação pela Ciência da Informação. Ciência da Informação, [S.I.], v. 42, n. 2, jan. 2015. Disponível em: <http://revista.ibict.br/ciinf/article/view/1382/1560>. Acesso em: 11 nov. 2016.

SILVA, Jonathas Luiz Carvalho; FREIRE, Gustavo Henrique. A. Um olhar sobre a origem da ciência da informação: indícios embrionários para sua caracterização identitária. Encontros Bibli: revista eletrônica de biblioteconomia e ciência da informação, v. 17, n. 33, p. 1-29, jan./abr. 2012.

SIMIONATO, Ana Carolina. Representação, acesso, uso e reuso da imagem digital. 141f. 2012. Dissertação (Mestrado em Ciência da Informação) - Universidade Estadual Paulista "Júlio de Mesquita Filho", Faculdade de Filosofia e Ciências, Marília/SP, 2012. p. 22-104.

SIMIONATO, Ana Carolina; SANTOS, Plácida L. V. Amorim da Costa. Descrição de recursos imagéticos digitais: apresentação de um modelo conceitual. Anales de Documentación, v. 16, n. 2, 2013. p.1-7. Disponível em: <http://revistas.um.es/analesdoc/article/view/179261/153931>. Acesso em 07 mar. 2017. 
SMITH, Mackenzie. Proposed: a 4-star classification-scheme for linked open cultural metadata. 2011. Disponível em: <http://lod-lam.net/summit/2011/06/06/proposeda-4-star-classification-scheme-for-linked-open-cultural-metadata/>. Acesso em 21 de mar. 2017.

TERSO, lole Costa. Alfabetização informacional: consciência e tecnologia na prática bibliotecária. In: SILVA, Rubens Ribeiro Gonçalves da et al (Org). Cultura, representação e informação digital. Salvador: Edufba, 2010. p. 99-118.

THORSEN, Hilary K.; PATTUELLI, M. Cristina. Linked Open Data and the cultural heritage landscape. In: JONES, Ed; SEIKEL, Michele (Eds.). Linked data for cultural heritage. Chicago: ALA Editions, 2016.

VOSS, Jon. Radically Open Cultural Heritage Data on the Web. In: MUSEUMS AND THE WEB, 2012. Anais... San Diego, CA, USA, 2012. Disponível em: <http://www.museumsandtheweb.com/mw2012/papers/radically_open_cultural_h eritage_data_on_the_w>. Acesso em 10 mar. 2017.

WORLD WIDE WEB CONSORTIUM. Semantic Web. 2015. Disponível em: <https://www.w3.org/standards/semanticweb/\#w3c_overview>. Acesso em 06 dez. 2016. 


\section{Apêndice A - Tabelas}

Tabela 1. A tipologia dos padróes de metadados

\begin{tabular}{|c|c|}
\hline $\begin{array}{c}\text { Tipologia dos padrões de } \\
\text { metadados }\end{array}$ & Exemplos \\
\hline $\begin{array}{l}\text { Padrões de estrutura de } \\
\text { dados }\end{array}$ & $\begin{array}{l}\text { O conjunto de campos do MARC (Machine-Readable Cataloging } \\
\text { format), Encoded Archival Description (EAD), Dublin Core Metadata } \\
\text { Element Set (DCMES), Categories for the Description of Works of Art } \\
\text { (CDWA), VRA Core Categories }\end{array}$ \\
\hline Padrões de valor de dados & $\begin{array}{l}\text { Library of Congress Subject Headings (LCSH), Library of Congress } \\
\text { Name Authority File (LCNAF), LC Thesaurus for Graphic Materials } \\
\text { (TCM), Medical Subject Headings (MeSH), Art \& Architecture } \\
\text { Thesaurus (AAT), Union List of Artist Names (ULAN), Getty Thesaurus } \\
\text { of Geographic Names (TCN), ICONCLASS }\end{array}$ \\
\hline $\begin{array}{l}\text { Padrões de conteúdo de } \\
\text { dados }\end{array}$ & $\begin{array}{l}\text { Anglo-American Cataloguing Rules (AACR), Resource Description and } \\
\text { Access (RDA), International Standard Bibliographic Description (ISBD), } \\
\text { Cataloging Cultural Objects (CCO), Describing Archives: Content } \\
\text { Standard (DACS) }\end{array}$ \\
\hline $\begin{array}{l}\text { Padrões de intercâmbio e } \\
\text { formato de dados }\end{array}$ & $\begin{array}{l}\text { MARC21, MARCXML, EAD XML DTD, METS, MODS, CDWA Lite XML } \\
\text { schema, Simple Dublin Core XML schema, Qualified Dublin Core XML } \\
\text { schema, VRA Core } 4.0 \text { XML schema }\end{array}$ \\
\hline
\end{tabular}

Fonte: Traduzido de GILLILAND, 2008, p. 3.

Tabela 2. Digitalização crítica vs. Digitalização em massa

\begin{tabular}{ll}
\hline \multicolumn{1}{c}{ Digitalização crítica } & \multicolumn{1}{c}{ Digitalização em massa } \\
\hline Essencialmente manual & Essencialmente automatizada \\
\hline $\begin{array}{l}\text { Reconhece as diferenças nas imagens que } \\
\text { podem ocorrer em cada objeto digitalizado }\end{array}$ & Trata a digitalização como um processo de clonagem \\
\hline $\begin{array}{l}\text { Realiza uma análise seletiva bem informada } \\
\text { dos objetos a serem digitalizados }\end{array}$ & $\begin{array}{l}\text { Normalmente escolhe os objetos elegíveis à } \\
\text { digitalização sem qualquer critério }\end{array}$ \\
\hline $\begin{array}{l}\text { Maximiza a interpretação e a elaboração de } \\
\text { metadados }\end{array}$ & $\begin{array}{l}\text { Minimiza a interpretação e a elaboração de } \\
\text { metadados }\end{array}$ \\
\hline $\begin{array}{l}\text { Adota critérios qualitativos sobre o que torna } \\
\text { um documento (ou conjunto de documentos) }\end{array}$ & $\begin{array}{l}\text { Adota critérios quantitativos sobre traços comuns, } \\
\text { regulares e previsíveis em um grande número de } \\
\text { documentos }\end{array}$ \\
\hline $\begin{array}{l}\text { único } \\
\text { Trata os documentos como artefatos gráficos, }\end{array}$ & $\begin{array}{l}\text { Foca-se nos textos dos documentos } \\
\text { espaciais e materiais }\end{array}$ \\
\hline Discriminativa: seleções / exibições & Exaustividade: exploração completa do acervo \\
\hline Profunda & Em escala \\
\hline
\end{tabular}

Fonte: Traduzido de Dahlström, 2010. 